\title{
A Phase I, multicenter, open-label trial to evaluate the safety of talimogene laherparepvec (T-VEC) injected into liver tumors
}

\author{
J Randolph Hecht ${ }^{1 *}$, Steven Raman ${ }^{1}$, Daniel Y Sze ${ }^{2}$, A Craig Lockhart ${ }^{3}$, Rebecca A Moss ${ }^{4}$, Kate Liu ${ }^{5}$, Jeffrey Chou ${ }^{5}$, \\ Tony Reid ${ }^{6}$
}

From 30th Annual Meeting and Associated Programs of the Society for Immunotherapy of Cancer (SITC 2015) National Harbor, MD, USA. 4-8 November 2015

\section{Introduction}

T-VEC, an intralesionally-delivered oncolytic immunotherapy, is a herpes simplex virus-1 engineered to selectively replicate in tumors and stimulate an antitumor immune response through expression of GMCSF. T-VEC has the ability to lyse various cancer cell types in vitro[1]. A Phase III study of T-VEC injected into skin, subcutaneous, or lymph node tumors versus subcutaneous GM-CSF in advanced melanoma demonstrated improved durable response rate for T-VEC, with regression of both injected and uninjected lesions[2]. To further explore if different types of cancers and locations might be treatable with T-VEC, this Phase I study evaluates whether primary and metastatic liver tumors may be safely and effectively injected with T-VEC.

\section{Methods}

Approximately 100 patients will be enrolled. Primary objective: evaluate maximum tolerated dose (MTD) of intrahepatic injection of T-VEC by patient incidence of dose-limiting toxicities (DLTs). Key secondary objectives: overall safety, efficacy, and biodistribution of T-VEC. Key eligibility criteria: breast, colorectal, gastroesophageal, kidney, lung cancer or melanoma with liver metastases (non-HCC) or hepatocellular carcinoma (HCC); measurable liver tumors suitable for injection; ECOG performance status $0-1$; life expectancy $\geq 5$ months; $\geq 1$ prior standard systemic anticancer therapy (non-HCC); ChildPugh A-B7; no detectable hepatitis B/C viral load; not a candidate for surgery or locoregional therapy of liver tumors with curative intent or planned systemic anticancer

'David Geffen School of Medicine at UCLA, Los Angeles, CA, USA Full list of author information is available at the end of the article therapy; tumor in $<1 / 3$ of the liver; no macroscopic intravascular invasion. The study consists of two parts. Part 1 : $3+3$ dose escalation of 3 sequential dose cohorts each administering T-VEC in increasing concentrations $\left(10^{7}\right.$ or $10^{8} \mathrm{PFU} / \mathrm{mL}$ ) and volumes (up to 4 or $8 \mathrm{~mL}$ ). MTD for $\mathrm{HCC}$ is determined separately from non-HCC tumor types; $\mathrm{HCC}$ cohorts will not proceed until safety at respective dose levels are determined in non-HCC. Six T-VEC doses injected under ultrasound or computed tomography guidance q21 $( \pm 3)$ days are planned, with an investigator option to continue for up to 6 additional doses. The first dose of T-VEC in all dose cohorts is given at $10^{6} \mathrm{PFU} / \mathrm{mL}$. Part 2: 7 expansion cohorts for each cancer type with 10 patients each administered the MTD of T-VEC determined from Part 1.

\section{Authors' details}

'David Geffen School of Medicine at UCLA, Los Angeles, CA, USA. ${ }^{2}$ Stanford University School of Medicine, Stanford, CA, USA. 'Washington University School of Medicine, St. Louis, MO, USA. ${ }^{4}$ Rutgers Cancer Institute of New Jersey, New Brunswick, NJ, USA. ${ }^{5}$ Amgen Inc., Thousand Oaks, CA, USA. ${ }^{6}$ Moores Cancer Center, University of California, San Diego, La Jolla, CA, USA.

Published: 4 November 2015

\section{References}

1. Liu BL, Robinson M, Han ZQ, et al: ICP34.5 deleted herpes simplex virus with enhanced oncolytic, immune stimulating, and anti-tumour properties. Gene Ther 2003, 10(4):292-303.

2. Andtbacka RH, Kaufman HL, Collichio F, et al: Talimogene Laherparepvec Improves Durable Response Rate in Patients with Advanced Melanoma. J Clin Oncol 2015, PubMed PMID: 26014293 [Epub ahead of print].

\section{doi:10.1186/2051-1426-3-S2-P180}

Cite this article as: Hecht et al:: A Phase I, multicenter, open-label trial to evaluate the safety of talimogene laherparepvec (T-VEC) injected into liver tumors. Journal for ImmunoTherapy of Cancer 2015 3(Suppl 2): P180. 